International Mathematical Forum, Vol. 9, 2014, no. 27, 1321 - 1332

HIKARI Ltd, www.m-hikari.com

http://dx.doi.org/10.12988/imf.2014.47139

\title{
Endo-prime Submodules
}

\section{in Endo-multiplication Modules}

\author{
Indah Emilia Wijayanti \\ Department of Mathematics \\ Universitas Gadjah Mada \\ Sekip Utara, Yogyakarta 55281, Indonesia
}

Copyright (c) 2014 Indah Emilia Wijayanti. This is an open access article distributed under the Creative Commons Attribution License, which permits unrestricted use, distribution, and reproduction in any medium, provided the original work is properly cited.

\begin{abstract}
There are various approaches to transfer the prime ideal notion to prime submodule. The definition of primeness in modules have appeared in many ways. In this paper we define endo-prime submodules and show that this definition and prime submodules introduced by Sanh et al. [8] are coincide. Moreover, we also present the endo-prime radical and its properties. Then we apply our results to the endo-multiplication modules, i.e. a multiplication module over its ring endomorphisms.
\end{abstract}

Mathematics Subject Classification: 16D50, 16D70, 16D80

Keywords: N-prime submodules, endo-prime submodules, endo-prime radicals, endo-multiplication modules

\section{Introduction}

Prime ideals, prime rings and radicals have an important role in a ring. Some previous authors have generalized those notions to modules. Since there are various approaches to transfer the prime ideal notion to prime submodule, the definition of primeness in modules have appeared in many ways. Wijayanti studied some notions of primeness of modules and one of them is endo-prime modules, i.e. the primeness of modules over its endomorphism ring (see [13]). 
In this paper we study further endo-prime submodules and show that the endo-prime submodules and prime submodules introduced by Sanh et al. [8] are coincide.

Furthermore, in a multiplication module one can define a multiplication between two submodules. Hence the observation of further properties of prime submodules can be done in a multiplication module. However, most of the previous paper presented multiplication modules over a commutative rings with unit (for example Ameri [1], Azizi [2], El-Bast [5] and [6], Tekir [10]). By the commutativity one can analyze the properties of prime submodules in a simple way. But if we generalize the commutative rings to the arbitrary rings, some properties will disappear, unless we add some condition. In this work we define a multiplication module over its endomorphism ring which is called an endo-multiplication module. Since an endomorphism ring is not necessary commutative, we refer to paper which studied a multiplication module over any ring (see for example Tuganbaev [12]). Moreover, we study the properties of endo-prime submodules and the radicals in an endo-multiplication module.

\section{N-Prime Submodules and Endo-prime Sub- modules}

In this section we give and study several definitions of primeness in a module. The first one is the definition of "prime submodules" introduced by Dauns [4], and we call it D-prime submodules. The second one is the definition of "prime submodules" by Sanh et.al [8], and we call it N-prime submodules. Furthermore we also recall the definition of "endo-prime submodules" by Wijayanti [13].

Throughout the paper, the ring means any ring with unit and the module means a right and unitair $R$-module. Moreover, we denote the endomorphism ring of $M$ by $S=\operatorname{End}_{R}(M)$. Consider that any right $R$-module $M$ is also a left $S$-module by defining $S \times M \rightarrow M,(f, n) \mapsto f(n)$ and sometimes we write as $f n$.

Definition 2.1 A submodule $N$ of a right $R$-module $M$ is called a D-prime submodule if for any $m \in M, r \in R$ and $m R r \subseteq N$, then $m \in N$ or $r \in$ $\operatorname{Ann}_{R}(M / N)$.

We refer to Theorem 1.3 of Dauns [4] to see the characterization of D-prime submodules. A submodule $K$ of $M$ is called fully invariant if for every $f \in$ $S, f(K) \subseteq K$. It is clear that as a left $S$-module, all its submodules are fully invariant. Conversely, any fully invariant submodule is also a left $S$ submodule. For any ideals $I, J \subset S$ and submodule $K \subset M$ we recall the 
following definition:

$$
\begin{aligned}
I(K) & =I K=\sum_{f \in I} f(K) \\
\operatorname{Ker}(I) & =\cap_{f \in I} \operatorname{Ker}(f) \\
I J & =\left\{\sum_{1 \leq i \leq n} f_{i} g_{i} \mid f_{i} \in I, g_{i} \in J, n \in \mathbb{N}\right\} .
\end{aligned}
$$

Now we recall from [8] the definition of N-prime submodule as follow.

Definition 2.2 Let $K$ be a fully invariant and proper submodule of $M$. Submodule $K$ is called an N-prime submodule in $M$ if for any fully invariant submodule invarian $L$ of $M$ and for every ideal $I$ of $S$, if $I(L) \subset K$, then $I(M) \subseteq K$ or $L \subset K$.

We refer to Theorem 1.2 of Sahn et al. [8] to give a characterization of an N-prime submodule. Now we give the definition of endo-prime submodules which is motivated by the definition of endo-prime modules in [13].

Definition 2.3 Let a right $R$-module $M$ and $S=\operatorname{End}_{R}(M)$. A fully invariant submodule $L$ in $M$ is called endo-prime submodule if for any fully invariant submodule $K / L$ in $M / L, \operatorname{Ann}_{S}(K / L)=\operatorname{Ann}_{S}(M / L)$. A module $M$ is called an endo-prime module if for any fully invariant $K, \operatorname{Ann}_{S}(K)=0$.

An endo-prime submodule and an endo-prime module are actually a Dprime submodule and a D-prime module over the endomorphism ring $S$ respectively. It is stated in the result of Wijayanti's observation in [13] as follow.

Proposition 2.4 $\quad$ (i) $M_{R}$ is an endo-prime module if and only if $M$ is D-prime as an $S$-module.

(ii) If $M_{R}$ is endo-prime, then $S$ is a prime ring.

Consider that statement (5) in Theorem 1.2 of Sahn et al. [8] and the definition of endo-prime submodule are equivalent, as we show in the following proposition.

Proposition 2.5 Let $L$ be a proper fully invariant submodule in $M$. The following assertions are equivalent:

a. $L$ is an endo-prime submodule.

b. $L$ is an N-prime $S$-submodule.

Proof. (a) $\Rightarrow$ (b) Take any $m \in M \backslash L$ and $f \in S$ which satisfy $f S m \subset L$. For any $m \in M \backslash L, S m$ is a fully invariant submodule which is not same with 
$L$. Hence $S m+L$ is a fully invariant submodule in $M$ which contains $L$, i.e. $L \subset S m+L$. So we obtain $(S m+L) / L$.

According to (a), $\operatorname{Ann}_{S}((S m+L) / L) \subseteq \operatorname{Ann}_{S}(M / L)$. It means whenever $f(S m+L) \subseteq L$, then $f(M) \subseteq L$. On the other words, if $f(S m) \subseteq L$, then $f \in \operatorname{Ann}_{S}(M / L)$.

(b) $\Rightarrow$ (a) Take any fully invariant submodule $K$ in $M$, where for any $f \in S, f(K) \subseteq L$. For any $n \in L, S n$ is a fully invariant submodule in $M$ and $f S n \subseteq L$. Thus $S n \subseteq K \subseteq M$. By assumption we conclude that $f \in \operatorname{Ann}_{S}$ or $f(M) \subseteq L . \diamond$

\section{Endo-Prime Radicals}

It is well known that in any ring $R$ we can define a multiplicatively closed subset $R^{\prime}$, i.e. a subset which contains no zero and for any $a, b \in R^{\prime}, a b \in R^{\prime}$. Lam [7] generalized this definition to m-system as follow. A non empty subset $R^{\prime} \subseteq R$ is called an m-system if for any $a, b$ in $S$ there is an $r$ in $R$ such that $a r b \in S$.

Now we recall a notion of m-system in module which was introduced by Behboodi [3] and Sanh et al. [9]. However, Sahn et al. gave a little modification to Behboodi's definition. For our purpose, we use the definition of Sanh et al. as we give below.

Definition 3.1 A non empty set $X \subseteq M \backslash\{0\}$ is called m-system if for any (left) ideal $A$ in $S$, any submodules $K, L$ in $M$ whenever $(K+L) \cap X \neq \emptyset$ and $(K+A M) \cap X \neq \emptyset$, then $(K+A L) \cap X \neq \emptyset$.

We present some results of Sanh et al. [9] related to the relationship between m-system and endo-prime submodule.

Lemma 3.2 (Lemma $6.2[9]$ ) Let $P$ be a proper fully invariant submodule in $M . P$ is endo-prime if and only if $M \backslash P$ is an m-system.

Next we show that any maximal submodule in $M$ is an endo-prime submodule if its intersection with any m-system in $M$ is empty.

Proposition 3.3 (Proposition 6.3 [9]) Let $X \subset M$ be an m-system and $P$ a maximal fully invariant submodule in $M$, where $P \cap X=\emptyset$. Then $P$ is an Endo-prime submodule.

Definition 3.4 Let $L$ be a fully invariant submodule in $M$. If there is an endo-prime submodule which contains $L$, then

$\operatorname{Rad}^{E}(L):=\{x \in M \mid$ for any $\mathrm{m}-$ system contains $x$ intersects with $L\}$. 
If there is no endo-prime submodule contains $L$, then $\operatorname{Rad}^{E}(L)=M$. The following proposition shows that $\operatorname{Rad}^{E}(L)$ is an intersection of all endo-prime submodules which contains $L$. We give first some notations :

$$
\begin{aligned}
V^{*}(L) & =\{P \in \operatorname{Spec}(M) \mid L \subset P\} ; \\
J\left(V^{*}(L)\right) & =\bigcap_{P \in V^{*}(L)} P .
\end{aligned}
$$

Proposition 3.5 (Proposition 6.5 [9]) Let L be a fully invariant submodule in $M$. Then $\operatorname{Rad}^{E}(L)=M$ or $\operatorname{Rad}^{E}(L)=J\left(V^{*}(L)\right)$.

As a special case in a ring $R$, if given an ideal $I$ and there exists a prime ideal which contains $I$, then as a corollary of Proposition $(3.5), \operatorname{Rad}^{E}(I)$ is the intersection of all ideals in $R$ which contains $I$ and denoted by $\sqrt{I}$. Otherwise, we define $\sqrt{I}=R$.

The further observation of the spectrum of prime submodules is in the frame of Zariski topology which is motivated by following papers : Yeşilot [14], Tekir [11] and Sanh et al. [9]. All endo-prime submodules of $M$, denoted by $\operatorname{Spec}^{E}(M)$ forms a topology called Zariski topology, where $V(N)$ is its closed set in the topology for any fully invariant submodule $N$ in $M$. We give the properties below.

Proposition 3.6 1. $V^{*}(0)=\operatorname{Spec}^{E}(M)$ and $V^{*}(M)=\emptyset$.

2. $\bigcap_{\lambda \in \Lambda} V^{*}\left(K_{i}\right)=V^{*}\left(\sum_{\lambda \in \Lambda} K_{i}\right)$ for any index set $\Lambda$.

3. $V^{*}(K) \cup V^{*}(L) \subseteq V^{*}(K \cap L)$ for any fully invariant submodules $K, L$ in $M$.

Proof. (1) and (2) are clear.

(3) Take any fully invariant submodules $K, L$ di $M$. By definition we have

$$
\begin{aligned}
V^{*}(K) & =\left\{P \in \operatorname{Spec}^{E}(M) \mid K \subseteq P\right\} ; \\
V^{*}(L) & =\left\{P \in \operatorname{Spec}^{E}(M) \mid L \subseteq P\right\} ; \\
V^{*}(K) \cup V^{*}(L) & =\left\{P \in \operatorname{Spec}^{E}(M) \mid K \subseteq P \text { or } L \subseteq P\right\} ; \\
V^{*}(K \cap L) & =\left\{P \in \operatorname{Spec}^{E}(M) \mid K \cap L \subseteq P\right\} .
\end{aligned}
$$

Take $P_{0} \in V^{*}(K) \cup V^{*}(L)$. It implies $P_{0}$ is an endo-prime submodule and satisfies $K \subseteq P_{0}$ or $L \subseteq P_{0}$. Then $K \cap L \subseteq K \subseteq P_{0}$ and $K \cap L \subseteq L \subseteq P_{0}$. Thus $P_{0} \in V^{*}(K \cap L)$. $\diamond$.

Especially, $\operatorname{Rad}^{E}(M)=J\left(V^{*}(0)\right)$. If $M$ contains an endo-prime submodule, then $\operatorname{Rad}^{E}(M)=\bigcap_{P \in \operatorname{Spec}^{E}(M)} P$. However, if there is no endo-prime submodules in $M$, then $\operatorname{Rad}^{E}(M)=M$. In this paper we assume the module $M$ contains an endo-prime submodule. 
Since $\operatorname{Rad}^{E}(M)$ is defined as the intersection of all endo-prime (fully invariant) submodules, then $\operatorname{Rad}^{E}(M)$ is also a fully invariant submodule in $M$ such that we obtain the factor module $M / \operatorname{Rad}^{E}(M)$. Then

$$
\operatorname{Rad}^{E}\left(M / \operatorname{Rad}^{E}(M)\right)=\bigcap_{\bar{P} \in \operatorname{Spec}^{E}\left(M / \operatorname{Rad}^{E}(M)\right)} \bar{P} .
$$

Before we give some further results of radicals, we present here some properties of endo-primeness related to factor module.

Lemma 3.7 Let $P$ be a proper fully invariant submodule in $M$ which contains $\operatorname{Rad}^{E}(M)$. If $\bar{P}=P / \operatorname{Rad}^{E}(M)$ is an endo-prime submodule in $M / \operatorname{Rad}^{E}(M)$, then $P$ is also an endo-prime submodule in $M$.

Proof. Take any $\varphi \in S, m \in M$ where $m \notin P$ and satisfies $\varphi S m \in P$.

Then $\bar{P}=P / \operatorname{Rad}^{E}(M)$ can be viewed as an $S$-module by the following scalar multiplication :

$$
\begin{aligned}
S \times P / \operatorname{Rad}^{E}(M) & \rightarrow P / \operatorname{Rad}^{E}(M), \\
(f, \bar{p})=\left(f, p+\operatorname{Rad}^{E}(M)\right) & \mapsto f(p)+\operatorname{Rad}^{E}(M)=f \bar{p}=\overline{f(p)} .
\end{aligned}
$$

For any $f \in S, \overline{\varphi f m}$ represents a class which contains $\varphi S m$ in $P / \operatorname{Rad}^{E}(M)$. Furthermore,

$$
\overline{\varphi f m}=\varphi f(m)+\operatorname{Rad}^{E}(M)=\varphi f \bar{m} \in P / \operatorname{Rad}^{E}(M) .
$$

Since $P / \operatorname{Rad}^{E}(M)$ is an endo-prime submodule in $M / \operatorname{Rad}^{E}(M)$,

$$
\varphi\left(M / \operatorname{Rad}^{E}(M)\right) \subseteq P / \operatorname{Rad}^{E}(M)
$$

or $\bar{m} \in P / \operatorname{Rad}^{E}(M)$. But $\bar{m} \notin P / \operatorname{Rad}^{E}(M)$, so

$$
\varphi\left(M / \operatorname{Rad}^{E}(M)\right)=(\varphi(M)) / \operatorname{Rad}^{E}(M) \subseteq P / \operatorname{Rad}^{E}(M) .
$$

We conclude that $\varphi(M) \subseteq P$. It means $P$ is an endo-prime submodule. $\diamond$

Lemma 3.8 Let $P_{1}$ and $P_{2}$ be proper fully invariant submodules in $M$ which contains $\operatorname{Rad}^{E}(M)$. Then

$$
\left(P_{1} / \operatorname{Rad}^{E}(M)\right) \cap\left(P_{2} / \operatorname{Rad}^{E}(M)\right)=\left(P_{1} \cap P_{2}\right) / \operatorname{Rad}^{E}(M) .
$$

Proof. Take any $\bar{x} \in\left(P_{1} / \operatorname{Rad}^{E}(M)\right) \cap\left(P_{2} / \operatorname{Rad}^{E}(M)\right)$. It implies $\bar{x} \in$ $\left(P_{1} / \operatorname{Rad}^{E}(M)\right)$ and $\bar{x} \in\left(P_{2} / \operatorname{Rad}^{E}(M)\right)$. Then

$$
\bar{x}=x_{1}+\operatorname{Rad}^{E}(M)=x_{2}+\operatorname{Rad}^{E}(M)
$$


and moreover $x_{1}-x_{2} \in \operatorname{Rad}^{E}(M)$ or on the other words $x_{1} \in P_{2} / \operatorname{Rad}^{E}(M)$ and $x_{2} \in P_{1} / \operatorname{Rad}^{E}(M)$. Thus $\bar{x} \in\left(P_{1} \cap P_{2}\right) / \operatorname{Rad}^{E}(M)$.

Conversely, take any $\bar{y} \in\left(P_{1} \cap P_{2}\right) / \operatorname{Rad}^{E}(M)$ which implies $\bar{y}=y_{0}+$ $\operatorname{Rad}^{E}(M)$, where $y_{0} \in P_{1} \cap P_{2}$. It is clear that $\bar{y} \in P_{1} / \operatorname{Rad}^{E}(M)$ and $\bar{y} \in$ $P_{2} / \operatorname{Rad}^{E}(M)$, so we prove that $\bar{y} \in\left(P_{1} / \operatorname{Rad}^{E}(M)\right) \cap\left(P_{2} / \operatorname{Rad}^{E}(M)\right)$. $\diamond$

Lemma 3.8 can be generalized to the family of fully invariant submodules in $M$ which contains $\operatorname{Rad}^{E}(M)$ as follow.

Corollary 3.9 Let $\left\{P_{\lambda}\right\}$ be the family of fully invariant submodules in $M$ which contains $\operatorname{Rad}^{E}(M)$. Then

$$
\bigcap_{\Lambda}\left(P_{\lambda} / \operatorname{Rad}^{E}(M)\right)=\left(\bigcap_{\Lambda} P_{\lambda}\right) / \operatorname{Rad}^{E}(M) .
$$

One of the important results in this work is the following

Proposition 3.10 $\operatorname{Rad}^{E}\left(M / \operatorname{Rad}^{E}(M)\right)=0$.

Proof. By definition we obtain

$$
\begin{aligned}
\operatorname{Rad}^{E}\left(M / \operatorname{Rad}^{E}(M)\right) & =\bigcap_{\bar{P} \in \operatorname{Spec}^{E}\left(M / \operatorname{Rad}^{E}(M)\right)} \bar{P} \\
& =\bigcap\left\{\bar{P}=P / \operatorname{Rad}^{E}(M) \mid P \text { submodule N }- \text { prima } \operatorname{di} M\right\} \\
& =\left(\bigcap_{P \in \operatorname{Spec}^{E}(M)} P\right) / \operatorname{Rad}^{E}(M) \\
& =\operatorname{Rad}^{E}(M) / \operatorname{Rad}^{E}(M)=0 . \diamond
\end{aligned}
$$

To investigate the properties of radical related to direct sums, we give below some prior results, that is for the direct sums of two submodules in $M$. For this purpose it is easy to understand that for any fully invariant submodules $K$ and $L$ in $M$ and $K \subseteq L$, then $\operatorname{Rad}^{E}(K) \subseteq \operatorname{Rad}^{E}(L)$.

Proposition 3.11 Let $K_{1}$ and $K_{2}$ be fully invariant submodules in $M$ and consider $K_{1} \oplus K_{2}$. Then

$$
\operatorname{Rad}^{E}\left(K_{1}\right) \oplus \operatorname{Rad}^{E}\left(K_{2}\right) \subseteq \operatorname{Rad}^{E}\left(K_{1} \oplus K_{2}\right) .
$$

Proof. Since $K_{1}, K_{2} \subseteq K_{1} \oplus K_{2}, \operatorname{Rad}^{E}\left(K_{1}\right), \operatorname{Rad}^{E}\left(K_{2}\right) \subseteq \operatorname{Rad}^{E}\left(K_{1} \oplus K_{2}\right)$. Then $\operatorname{Rad}^{E}\left(K_{1}\right) \oplus \operatorname{Rad}^{E}\left(K_{2}\right) \subseteq \operatorname{Rad}^{E}\left(K_{1} \oplus K_{2}\right)$.

For converse of Proposition (3.11) we need some condition as we show in the next proposition.

Proposition 3.12 Let $M$ be a module whose every fully invariant submodule is endo-prime. If $K_{1}$ and $K_{2}$ are fully invariant submodules in $M$, then

$$
\operatorname{Rad}^{E}\left(K_{1}\right) \oplus \operatorname{Rad}^{E}\left(K_{2}\right)=\operatorname{Rad}^{E}\left(K_{1} \oplus K_{2}\right) .
$$


Proof. It is sufficient to show that $\operatorname{Rad}^{E}\left(K_{1} \oplus K_{2}\right) \subseteq \operatorname{Rad}^{E}\left(K_{1}\right) \oplus \operatorname{Rad}^{E}\left(K_{2}\right)$. Assume $x \notin \operatorname{Rad}^{E}\left(K_{1}\right) \oplus \operatorname{Rad}^{E}\left(K_{2}\right)$. Without loss of the generality, $\pi_{1}(x)=$ $x_{1} \notin \operatorname{Rad}^{E}\left(K_{1}\right)$. So there is an endo-prime submodule $Y$ which contains $K_{1}$ but does not contain $x_{1}$. Then we form fully invariant submodule $Y \oplus X_{2}$ where $X_{2}$ is an endo-prime submodule which contains $K_{2}$. Since every fully invariant submodules in $M$ is endo-prime, $Y \oplus X_{2}$ is endo-prime submodule and contains $K_{1} \oplus K_{2}$ bit does not contain $x$. Hence $x \notin \operatorname{Rad}^{E}\left(K_{1} \oplus K_{2}\right)$. We prove that $\operatorname{Rad}^{E}\left(K_{1} \oplus K_{2}\right) \subseteq \operatorname{Rad}^{E}\left(K_{1}\right) \oplus \operatorname{Rad}^{E}\left(K_{2}\right)$.

Corollary 3.13 Let $M$ be a module whose every fully invariant submodule is endo-prime. Let $\left\{K_{\lambda}\right\}_{\Lambda}$ be the family of fully invariant submodules in $M$. Then

$$
\bigoplus_{\Lambda} \operatorname{Rad}^{E}\left(K_{\lambda}\right)=\operatorname{Rad}^{E}\left(\bigoplus_{\Lambda} K_{\lambda}\right)
$$

\section{Endo-multiplication Modules}

To accommodate the definition of endo-prime submodule which includes endomorphisms ring, in this work we define a multiplication module over its endomorphisms ring.

Definition 4.1 A module $M$ is called an endo-multiplication modules if for any fully invariant submodule $L$ in $M$ there is an ideal $T$ in $S$ such that $L=T M$.

We modify Lemma 2.4 of Ameri [1] for any ring, especially endomorphisms ring

Proposition 4.2 Let $M$ be an endo-multiplication module and $L$ a fully invariant submodule in $M$. Then $L=\operatorname{Ann}_{S}(M / L) M$.

Proof. Let $L$ be a fully invariant submodule in $M$. Since $M$ is an endomultiplication module, then there exists an ideal $T$ in $S$ such that $L=T M$. Take any $f \in T$ and $m+L \in M / L$, then we have $f(m+L) \subseteq L$. It means $f \in \operatorname{Ann}_{S}(M / L)$ and $L \subseteq \operatorname{Ann}_{S}(M / L) M$.

Conversely, for any $f \in T, m \in M$ and $f(m) \in L$ will implies $\operatorname{Ann}_{S}(M / L) \subseteq$ L. $\diamond$

We also compare the endo-multiplication modules with multiplication modules over $S$ as we show in the following proposition.

Proposition 4.3 The following assertions are equivalent:

a. $M$ is a multiplication S-module;

b. $M$ is an endo-multiplication module. 
Proof. (a) $\Rightarrow$ (b) Take any fully invariant submodule $L$ in $M$, then for any $I \subseteq S$ we have $I(L) \subseteq L$. Hence $L$ is an $S$-submodule. Since $M$ is an $S$-modul multiplication, there exists an ideal $T$ in $S$ such that $L=T M$.

(b) $\Rightarrow$ (a) Take any $S$-submodule $L$ in $M$, then for any $I \subseteq S$ we have $I(L) \subseteq L$. Hence $L$ is a fully invariant submodule in $M$. Since $M$ is an endomultiplication module, then there exists an ideal $T$ in $S$ such that $L=T M$. $\diamond$

Furthermore, the converse of (3) in Proposition (3.6) will be true if the module $M$ is an endo-multiplication module as we give in the next

Corollary 4.4 If $M$ is an endo-multiplication module, then for any fully invariant submodule $K$ and $L, V^{*}(K) \cup V^{*}(L)=V^{*}(K \cap L)$.

Proof. It is sufficient to show $V^{*}(K \cap L) \subseteq V^{*}(K) \cup V^{*}(L)$. Take any $P \in V^{*}(K \cap L)$. It implies $P$ an endo-prime submodule and $K \cap L \subseteq P$. It easy to understand that $K L=\operatorname{Ann}_{S}(M / K) \operatorname{Ann}_{S}(M / L) M \subseteq K \cap L \subseteq P$. Since $P$ is an endo-prime submodule and applying Proposition (4.7), $K \subseteq P$ or $L \subseteq P$. Hence $P \in V^{*}(K) \cup V^{*}(L)$. $\diamond$

A direct consequence of Corollary (4.4) is the following

Corollary 4.5 If $M$ is an endo-multiplication module, then for any fully invariant submodules $K$ and $L, \operatorname{Rad}^{E}(K \cap L)=\operatorname{Rad}^{E}(K) \cap \operatorname{Rad}^{E}(L)$.

\section{Proof.}

$$
\begin{aligned}
\operatorname{Rad}^{E}(K \cap L) & =J\left(V^{*}(K \cap L)\right)=J\left(V^{*}(K) \cup V^{*}(L)\right) \\
& =J\left(V^{*}(K)\right) \cap J\left(V^{*}(L)\right)=\operatorname{Rad}^{E}(K) \cap \operatorname{Rad}^{E}(L) . \diamond
\end{aligned}
$$

Now we give a necessary and sufficient condition for an endo-multiplication module.

Proposition 4.6 The following assertions are equivalent:

a. $M$ is an endo-multiplication modules;

b. for any fully invariant submodule $L$ in $M L=\operatorname{Ann}_{S}(M / L) M$;

c. for any $m \in M$ there exists an ideal $I$ in $S$ such that $S m=I M$.

Proof. (a) $\Rightarrow$ (b) It is clear from Proposition (4.3).

(b) $\Rightarrow$ (c) For any $m \in M, S m$ is a fully invariant submodule in $M$. From assumption in (b), $S m=\operatorname{Ann}_{S}(M / S m) M$. Hence there exists $I=$ $\operatorname{Ann}_{S}(M / S m)$ such that $S m=I M$.

(c) $\Rightarrow$ (a) Take any fully invariant submodule $L$ in $M$. From assumption (c), for any $n \in L, S n=I M$ for some ideal $I$ in $S$. Hence $L \subseteq I M$. On the other hand, since $L$ is fully invariant, $I M=S n \subseteq L$. So $L=I M$. $\diamond$ 
We know that the presentation ideal for each submodule in an endo-multiplication module is not unique. This fact will influence the definition of the multiplication between two submodules. For the special case, that is multiplication module over a commutative ring, the definition between two submodules is well defined. (see for example paper of Ameri [1] or Azizi [2]).

By using Proposition (4.6) we define the multiplication between two submodules as follow. According to Proposition (4.6), there exist ideals $\operatorname{Ann}_{S}(M / K)$ and $\operatorname{Ann}_{S}(M / L)$ such that $K=\operatorname{Ann}_{S}(M / K) M$ and $L=\operatorname{Ann}_{S}(M / L) M$. We define the multiplication between $K$ and $L$ as follow.

$$
K L:=\operatorname{Ann}_{S}(M / K) \operatorname{Ann}_{S}(M / L) M .
$$

It is clear that the multiplication in (1) is unique. If this invariant submodule is cyclic, $K=<k>$ and $L=<l>$, then

$$
k . l:=<k><l>=\operatorname{Ann}_{S}(M /<k>) \operatorname{Ann}_{S}(M /<l>) M .
$$

Now we give some results related to the multiplication between submodules and the primeness.

Proposition 4.7 Let $M$ be an endo-multiplication module, $K, L$ and $P$ fully invariant submodules in $M . P$ is an endo-prime submodule if and only if whenever $K L \subseteq P$, then $K \subseteq P$ or $L \subseteq P$.

Proof. $(\Rightarrow)$ Take any fully invariant submodule $K$ and $L$ and satisfy $K L \subseteq P$. Then

$$
K L=\operatorname{Ann}_{S}(M / K) \operatorname{Ann}_{S}(M / L) M \subseteq P .
$$

Since $P$ is an N-prime submodule, $\operatorname{Ann}_{S}(M / K) M=K \subseteq P$ or $\operatorname{Ann}_{S}(M / L) M=$ $L \subseteq P$.

$(\Leftarrow)$ Take any $\varphi \in S, m \in M \backslash P$ and satisfies $\varphi S m \in P$. Hence $\varphi M \nsubseteq P$. It implies $m_{0} \in M$ where $\varphi m_{0} \notin P$. Then we build endo-prime submodules $<m_{0}>=S m_{0}$ and $<\varphi m>=S \varphi m$. Since $M$ is an endo-multiplication module, there exists ideal $T, U$ in $S$ such that $S m_{0}=T M$ and $S \varphi m=U M$. Now we present

$$
\left(S m_{0}\right)(S \varphi m)=T M U M=T U M \subseteq U M=S \varphi m \subseteq P .
$$

For Assumption we have $S m_{0} \subseteq P$ or $S \varphi m \subseteq P$. Thus $m_{0} \in P$ or $m \in P$, a contradiction. We prove that $P$ is an endo-prime submodule. $\diamond$

As a special case of Proposition (4.7), i.e. if the submodules are cyclic in $M$, then we have the following

Corollary 4.8 Let $M$ be an endo-multiplication module and $P$ a proper fully invariant submodule in $M . P$ is an endo-prime submodule if and only if for any $m_{1}, m_{2} \in M, m_{1} . m_{2} \subseteq P$ then $m_{1} \in P$ or $m_{2} \in P$. 
Proof. $(\Rightarrow)$ It is clear.

$(\Leftarrow)$ Take any fully invariant submodules $M_{1}$ and $M_{2}$ where $M_{1} M_{2} \subseteq P$. Assume $M_{1} \nsubseteq P$ and $M_{2} \nsubseteq \nsubseteq P$, there exist $m_{1} \in M_{1} \backslash P$ and $m_{2} \in M_{2} \backslash P$. We obtain

$$
m_{1} \cdot m_{1} \subseteq M_{1} M_{2} \subseteq P .
$$

But we know that $m_{1} \in P$ or $m_{2} \in P$, a contradiction with the assumption. Hence according to Proposition (4.7), $P$ is an endo-prime submodule. $\diamond$ In the last section, we give a general situation of Nakayama Lemma in module.

Definition 4.9 Element $u$ in $M$ is called a unit element if $u$ is not contained in any maximal submodules.

A characterization of a unit in an endo-multiplication module is given below.

Proposition 4.10 Let $M$ be an endo-multiplication module. $u$ is a unit in $M$ if and only if $\langle u\rangle=M$.

Proof. $(\Leftarrow)$ It is obvious.

$(\Rightarrow) u$ is a unit in $M,\langle u\rangle$ is an ideal which is contained $u$ and is not contained in any maximal submodule. Hence $\langle u\rangle=M$. $\diamond$

Proposition 4.11 Assume the module $M$ contains a unit $u . m \in \operatorname{Rad}^{E}(M)$ if and only if $u-g m$ is a unit in $M$ for any $g \in S$.

Proof. $(\Rightarrow)$ Let $m \in \operatorname{Rad}^{E}(M)$. Applying Proposition (4.10), it is showed that $\langle u-g m\rangle=M$. It is clear that $\langle u-g m\rangle \subseteq M$ and sufficient to prove that $M \subseteq<u-g m>$. Assume $M \nsubseteq<u-g m>$. There exists $m_{0} \in M$ and $m_{0} \notin<u-g m>$. Hence $m_{0} \notin S u+S g m$ or equivalently $m_{0} \notin M+S g m=M$ or $m_{0} \notin M$, a contradiction.

$(\Leftarrow)$ Let $\left\langle u-g m_{0}\right\rangle=S u+S g m_{0}=M$. Assume $m_{0} \notin \operatorname{Rad}^{E}(M)$. There exists an endo-prime submodule $P$ in which for any $f \in S$ where $f S m_{0} \subseteq P$, $m_{0} \notin P$ but $f(M) \nsubseteq P$. Consider that

$$
\begin{aligned}
f(M) & =f\left(S u+S g m_{0}\right)=f(S u)+f\left(S g m_{0}\right) \\
& =f(M)+f\left(S g m_{0}\right) .
\end{aligned}
$$

Thus $f\left(S g m_{0}\right) \subseteq f(M)$ for any $g \in S$. We conclude that $f\left(S m_{0}\right) \subseteq f(M) \nsubseteq P$. A contradiction to $f S m_{0} \subseteq P$. $\diamond$

Acknowledgements. The author would like to thank to the referees. This work was supported by Research Grant No. 36/J01.1.28/PL.06.02/2012 by Department of Mathematics, Universitas Gadjah Mada, Yogyakarta, Indonesia. 


\section{References}

[1] R. Ameri, On the prime submodules of multiplication modules, IJMMS Vol. 27 (2003), 1715 - 1724.

[2] A. Azizi, Radical formula and prime submodules, Journal of Algebra 307 (2007), $454-460$.

[3] M. Behboodi, On the Prime Radical and Baer's Lower Nilradical of Modules, Acta Math. Hungar. 122 (3) (2009), 293-306.

[4] J. Dauns, Prime Modules, Journal für die reine und angewandte Mathematik 298 (1978), 156 - 181.

[5] Z.A. El-Bast and P.F. Smith, Multiplication Modules, Communications in Algebra 16(4) (1988), 755 - 779.

[6] Z.A. El-Bast and P.F. Smith, Multiplication Modules and Theorems of Mori and Mott, Communications in Algebra 16(4) (1988), 781 - 796.

[7] T.Y. Lam, A First Course in Noncommutative Rings, Springer, 1991.

[8] N. V. Sanh, N. A.Vu, K. F. U. Ahmed, S. Asawasamrit, L. P. Thao, Primeness in Module Category, Asian-European Journal of Mathematics Vol. 3 no. 1 (2010), 145-154.

[9] N.V. Sanh, L.P. Thao, N.F.A. Al-Mayahi, K.P. Shum, Zariski Topology of Prime Spectrum of a Module, Proceedings of the International Conference on Algebra 2010, World Scientific, 2012.

[10] U. Tekir, On Multiplication Modules, International Mathematical Forum Vol. 2 No. 29 (2007), 1415 - 1420.

[11] U. Tekir, The Zariski Topology on the Prime Spectrum of a Module over Noncommutative Rings, Algebra Colloquium Vol. 16 No. 4 (2009), 691 698.

[12] A.A. Tuganbaev, Multiplication modules over non-commutative rings, Mathematicheskii Sbornik Vol. 194, No. 12 (2003), 1837 - 1864.

[13] I.E. Wijayanti, Coprime Modules and Comodules, Dissertation, University of Düsseldorf, Germany (2006).

[14] G. Yeşilot, On the Prime Spectrum of a Module over Noncommutative Rings, International Journal of Algebra Vol. 5 No. 11 (2011), 523 - 528.

Received: July 28, 2014 